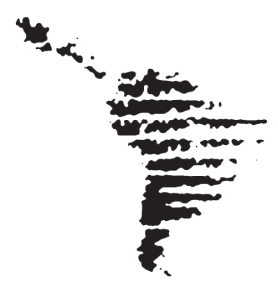

\title{
La configuración de la juridicidad y antijuridicidad del daño ambiental y sus diferentes formas de legitimidades ${ }^{1}$
}

\section{Shaping legality and illegality of environmental damage and its different ways of legitimacy}

Claudia Alexandra Munévar Quintero²

\section{Resumen}

El presente artículo tiene como marco de análisis la configuración del daño ambiental desde sus diferentes formas de legitimidades. El objetivo es develar el contexto constitucional y doctrinal del daño en relación con el ambiente mismo, según su carácter jurídico y anti-jurídico y la relación con víctimas determinadas o indeterminadas, titular de derechos ambientales. En este sentido, mediante la técnica de análisis documental se esbozan los postulados normativos del "ser" y "deber ser" de la norma. Para ello, se parte de la comprensión que el daño, más que una construcción jurídica, tiene una definición social, la cual, a su vez, determina los criterios de juridicidad. Se concluye que este criterio de juridicidad no atiende solamente juicios de razonabilidad, fuerza y poder de un Estado a través de sus normas, sino que son establecidas según los consensos y las re-significaciones sociales. Ampararse en la coacción del derecho y su fuerza arbitraria conlleva a la deslegitimación de las actuaciones legales de un Estado y acentúa la brecha entre la realidad normativa y las realidades sociales.

Palabras clave: Daño ambiental, daño jurídico, daño antijurídico, legitimidad.

\begin{abstract}
This article has as a configuration framework wich concerns the environmental damage from the different forms of legitimacy. The aim is to uncover the constitutional and doctrinal context of damage related with the environment according to their legal and anti-legal nature; it can be seen as a determinate or indeterminate victim, which is the owner of environmental rights. The epistemological approach is hermeneutical, in this sense, the normative postulates of

$1 \quad$ El artículo se deriva del proyecto de investigación: La legitimidad de las licencias ambientales y su incidencia en la vulneración de derechos colectivos e intergeneracionales, en el contexto de conflictos socio-ambientales en Colombia. Esta investigación corresponde a una tesis doctoral para optar por el título de Doctora en Desarrollo Sostenible de la Universidad de Manizales Colombia.

2 Abogada-docente-investigadora- Universidad de Manizales, Colombia. Especialista en Derecho Administrativo- Universidad de Caldas. Magíster en Desarrollo Sostenible y Medio Ambiente- Universidad de Manizales. Estudiante Doctorado en Desarrollo Sostenible.
\end{abstract}


"being" and "should be" of the regulations are outlined through the documentary analysis technique. To do this, it is understood that the damage, more than a legal construction, has a social definition, which in turn determines the criteria of legality. It is concluded that this criterion of legality does not serve only to judgments of a State's reasonableness, strength and power through its rules. On the other hand, they are established by consensus and social re-significances. Relying on the coercion of law and its arbitrary force, leads to the discrediting of the State's legal proceedings, accentuating the gap between the normative reality and social realities.

Keywords: environmental damage, legal damage, illegal damage, legitimacy.

\section{Introducción}

En el contexto de la legalidad de un Estado para determinar el daño ambiental producido en el marco de proyectos y obras de desarrollo, el derecho se ha convertido en uno de los determinantes de presunción de legitimidad que justifica no solo el impacto significativo sobre el medio ambiente y los recursos naturales, sino además las vulneraciones en los derechos humanos tanto de naturaleza individual como colectiva. Sin duda se trata de daños evidentes del mundo actual; no obstante, frente a su evidencia, han surgido nuevas categorías teóricas y legales que problematizan el reconocimiento y reparación del daño ambiental, sea de naturaleza pura o consecutiva. Como se anotará en el desarrollo de este artículo, existe un elemento denominado la juridicidad del daño, componente que define los criterios para el reconocimiento de este, en el sentido de establecer si ese daño se debe o no soportar. Frente a este último aspecto se generará el debate y marco de análisis de este artículo, por cuanto, qué o quién determina si se está en el deber jurídico de soportar ese daño o no.

Recurrir a la teoría política pareciera una salida importante al momento de encontrar definiciones sobre la naturaleza de la legitimidad;legitimidad en el contexto de la "autorización" por parte de un Estado o de las leyes, para que un individuo, una colectividad o el ambiente en sí mismo deban soportar un daño. No obstante, pese a que las definiciones son puntuales frente a la definición del concepto, no presenta soluciones a la evidente vulneración de derechos "permitidos" desde el manto de la legalidad.

Por esta razón, el presente artículo pone en evidencia otras connotaciones y conceptos frente a la legitimidad social. Al mismo tiempo, estas definiciones de legitimidad como forma de consenso social cuestionan los fundamentos doctrinales e interpretativos del concepto de juridicidad y permiten concluir que la legitimidad del Estado actual no debe enmarcarse en el uso de la fuerza o en el amparo de coacción, sino en la capacidad de identificar sus postulados con las realidades $y$ necesidades sociales, así como en las 
diferentes valoraciones y percepciones de los miembros de una comunidad o de la sociedad en general, respecto a la consideración del daño ambiental.

\section{La configuración de la juridicidad y antijuridicidad del daño ambiental}

La concepción del daño como elemento y presupuesto que establece el deber jurídico de la reparación ha reconfigurado el régimen de responsabilidad civil en Colombia desde diferentes aspectos. Por un lado, su fundamento no solo radica en atender a criterios de concepciones que solo tengan en cuenta la culpa, sino que su reparación actualmente abarca criterios y dimensiones de justicia y equidad, "es así como la prioridad no es buscar un culpable para sancionarlo, sino comprender y reparar a la víctima por el mal injustamente sufrido" (Gil, 2011, p. 24).

De igual forma, estas reconfiguraciones han dadolugar a nuevas comprensiones en el ámbito del derecho que, en aras de garantizar justicia y equidad, han roto paradigmas dogmáticos y principios jurídicos antropocéntricos que parecían ser inquebrantables, dando lugar a la protección de derechos como los colectivos ambientales y el reconocimiento y reparación de daños que componen esta misma dimensión. Es así como aparecen diferentes clasificaciones que trascienden del reconocimiento de daños que afectan la esfera patrimonial de un individuo, al reconocimiento de daños, que tienen una naturaleza "supraindividual", la cual involucra no solo sujetos determinados, sino colectividades determinadas y no determinadas.

Nos adelantamos en señalar las características jurídicas del daño ambiental: no es un daño común, es ambivalente o bifronte en cuanto afecta intereses individuales y supraindividuales, es complejo, de relación causal difusa, vinculado con aspectos técnicas o científico tecnológicos, de ardua, costosa o difícil comprobación, en ocasiones anónimo o impersonal, prevalentemente social, colectivo o masificado. A su vez constituye el único daño civil constitucionalizado. (Caferrata, 2004, p. 63)

En este sentido, la doctrina ha entrado a distinguir entre el daño que se genera al ambiente propiamente dicho y el daño que genera consecuencias a las esferas patrimoniales y morales de un individuo o una colectividad. Sobre el primer sentido, Rojas (2012) define el perjuicio ambiental propiamente dicho como aquel que "... recae sobre el derecho colectivo al ambiente sano y que, en esa medida, en principio, no se materializa sobre una víctima individualizada ..." (p. 80). Este tipo de daño lo define Henao (2000) como daño ambiental puro:

Aquello que ha caracterizado regularmente las afrentas al medio ambiente es que no afectan especialmente a una 
$\mathrm{u}$ otra persona determinada, sino exclusivamente el medio natural en sí mismo considerado; es decir, las cosas comunes, que en ocasiones hemos designado como bienes ambientales, tales como el agua, el aire, la flora y la fauna salvaje. (Henao, 2000, p. 143)

En segundo sentido, el daño que genera consecuencias individuales es definido como daño ambiental impropio o daño consecutivo, "se trata de daños consecuenciales.... No es ya una lesión del interés colectivo del ambiente mismo, sino el menoscabo patrimonial o extrapatrimonial padecido por un individuo, como consecuencia o reflejo de la contaminación ambiental” (Rojas, 2012, p. 84).

Es así como estas distinciones han dado lugar a un reconocimiento importante en materia de daño ambiental, toda vez que la dimensión en el daño permite conjugar dos tipos de perjuicios: "el perjuicio que sufren todos, es decir la humanidad, pues se está alterando algo que es de todos... y el perjuicio que sufre el titular de la propiedad privada sobre el medio ambiente afectado, pues su bien se dañó” (González, 2007, p. 230).

Ahora bien, para el reconocimiento y reparación del daño, independientemente a qué tipo de daño se refiera, sea puro o consecutivo, se requiere de otro elemento: este es que el daño sea antijurídico. Este elemento tiene una importante connotación en el marco jurídico y constitucional colombiano, toda vez que el artículo 90 de la Constitución Política de 1991 alude a este término como criterio de responsabilidad: "El Estado responderá patrimonialmente por los daños antijurídicos que le sean imputables, causados por la acción o la omisión de las autoridades públicas. [En este sentido,] ¿cuándo es jurídico el daño? La respuesta es clara: cuando existe el deber legal de soportarlo" (Gil, 2011, p. 28).

Esta concepción o definición de daño jurídico antijurídico da a entender que en la esfera de la reparación de un daño hay que considerar si su causa es jurídica o no lo es, es decir, que la ley o el actuar legítimo de un Estado no lo imponga.

Y no deben soportarse daños, independientemente de que sean la consecuencia del obrar legítimo o del actuar contrario al orden jurídico, cuando no exista razón legal o de derecho que obligue a padecerlos, porque, de lo contrario, el daño es antijurídico para quien lo sufre, en la medida en que el ordenamiento no se lo impone. (Gil, 2011, p. 28)

En materia ambiental esta concepción de daño tiene una connotación importante, toda vez que el medio ambiente en Colombia es concebido y definido como un recurso natural, recurso objeto de utilización, 
aprovechamiento y explotación, bajo el expuesto y el entendido de que dichas obras sean "racionales", y dicho criterio de racionalidad se delega al Estado a través del mandato constitucional y legal.

Entonces, si se hace uso o aprovechamiento de los recursos naturales renovables cumpliendo con los requisitos normativos, esto es, usándolos dentro de los niveles, cantidades $\mathrm{y}$ concentraciones, y con las condiciones impuestas en el acto administrativo que permite su uso o aprovechamiento, el daño fatal que de todas formas se presente es un daño jurídico, lícito y permitido. (González, 2007, p. 231)

Desde esa óptica, es el marco jurídico el que delimita la juridicidad y antijuridicidad del daño, de ahí que se considere ilícito todo lo que exceda los límites permisibles trazados para dicho aprovechamiento y explotación y, por ende, la consideración de justo o injusto del daño soportado por una víctima lo establece la norma. Ahora bien, el criterio de justo o injusto resulta problemático, más aún cuando su valoración versa sobre derechos humanos. Por ende, desde la perspectiva de legalidad, mas no de legitimidad, es más conveniente "acudir no la calificación de justicia o injusticia del daño, sino más bien al concepto de antijurídico ..." (Gil, 2011, p. 27).

Esta distinción de daño jurídico y daño antijurídico o daño lícito o ilícito es útil en el marco del aprovechamiento de la oferta ambiental, toda vez que, en el ámbito normativo colombiano, el ambiente es concebido como recurso, es decir, "objetos puestos a nuestra disposición y con un valor económico, como único valor" (Noguera, 2004, p. 66). No obstante, una cosa es la oferta ambiental que puede ser explotada y otra es un derecho ambiental que puede ser vulnerado. En el ámbito de derechos ambientales, cuyo alcance y reconocimiento son de índole humana, el concepto de juridicidad no es apto, toda vez que su vulneración, sí, categóricamente sí, debe responder a criterios de justicia. El cuestionamiento no se eleva al nivel de la juridicidad o antijuridicidad, sino al nivel de legitimidad de un Estado, el cual no solo considera como jurídico un daño, sino la vulneración de un derecho desde su naturaleza fundamental o colectiva, pero ambos de índole humana.

\section{El daño ambiental y sus diferen- tes formas de legitimidades}

El concepto de Estado se encuentra asociado con el tema de "poder y dominación" (Weber, 1964), toda vez que sus prácticas políticas versan sobre esta noción. Ahora bien, el 
poder, además de ser un concepto polisémico, es amorfo, toda vez que "las cualidades imaginables de un hombre y toda suerte de constelaciones posibles pueden colocar a alguien en la posición de imponer su voluntad en una situación dada" (Weber, 1964, p. 43). El poder encuentra su justificación en formas de dominación que pueden ser justas o injustas, pero en todo caso son vinculantes; en este sentido “... el poder es la capacidad de tomar decisiones formales que de algún modo son vinculantes" (Heywood, 2010, p. 148).

Este ejercicio legítimo de poder ha sido monopolizado por el Estado, el cual es entendido como "un instituto político de actividad continuada, cuando y en la medida en que su cuadro administrativo mantenga con éxito la pretensión al monopolio legítimo de la coacción física para el mantenimiento del orden vigente" (Weber, 1964, p. 43). En la práctica del ejercicio del poder, el Estado no solo se reservó el monopolio de la coacción física, sino el monopolio de lo cierto y de lo falso, de lo justo y de lo injusto y así de lo legítimo e ilegítimo. Por ende, no solo es el monopolio del hacer, sino del saber, el cual, según sus propios juicios y por medio de sus funciones, en principio legislativas y ejecutivas, todo lo que esté fuera de los parámetros señala- dos es inexistente o, en términos jurídicos, ilegal: "todo lo que el canon no legitima o no reconoce es declarado inexistente" (Sousa, 2010, p. 42).

Una de las formas de materialización de la legitimidad por parte de un Estado es a través de sus mandatos, cuyos instrumentos son sus normas o convenciones jurídicas, por medio de las cuales "un legislador se encuentra autorizado a promulgar esa ley como una norma...Aquí, la legitimación es un proceso por el cual un legislador... está autorizado a prescribir las condiciones convenidas" (Lyotard, 2012, p. 23). Estas convenciones no necesariamente fundadas en acuerdos o necesidades sociales y cuya mayor y principal arma de mando es el ejercicio del poder. Esta agudeza del ejercicio del derecho ha llevado a que su fundamento y noción de validez sea comprendida desde el principio de legalidad y que este concepto sea erróneamente asimilado con el de legitimidad.

Todo el Estado es de derecho y todo derecho es del Estado- es una de las grandes innovaciones de la modernidad occidental... Es también una simetría muy problemática ... porque afirma la autonomía del derecho con relación a lo político en el mismo proceso en que hace depender su validez del Estado. (Sousa, 2010, p. 106) 
Precisamente es desde estas comprensiones de legitimidad que se ha dado lugar al concepto de juridicidad y antijuridicidad; no desde lo justo y lo injusto, sino de lo legal e ilegal. Este ejercicio de legitimidad pretende que sea el juicio de un Estado el que determine si una persona o población tiene el deber jurídico de soportar un daño ambiental, sea puro o consecutivo; lo importante es que ese perjuicio sea legal en la medida que es jurídico.

Queda claro cómo en materia de responsabilidad por daño ambiental y dentro de este marco de interpretación no basta el criterio de la simple comisión de un daño, sino que su deber de reparar se fundamente en la juridicidad de este mismo. En este sentido y en este contexto, la antijuridicidad de un daño no se traduce en "aquel que la víctima no está obligada a soportar", sino en aquel daño que el Estado, por medio de sus marcos normativos, establece qué tipo de daño una víctima debe soportar, toda vez que en el ejercicio de su poder legítimo del hacer y el "saber", podría establecerlo, sin que la víctima pueda refutarlo o que, al hacerlo, dicha reparación sea nula o insuficiente, por demás injusta, aunque legal. Se trata, entonces, de una legitimidad de quienes gobiernan, que, sin considerar a los sujetos gobernados "no se compromete la responsabilidad del individuo cuando, a pesar de haber sido irrogado un daño, éste está autorizado por un sistema jurídico" (Rojas, 2012, p. 101). Supuestamente, como lo menciona el mismo autor (Rojas, p.103): "el gasto de oferta ambiental es permitido, siempre que se circunscriba a unos cánones de razonabilidad", los cuales se inscribieron en la legitimidad del Estado investidos de la fuerza de la coerción.

La legitimidad de la coerción implica que el agente que la ejerce le niega a su objeto el derecho a resistirse a esa coerción, a cuestionar sus motivos, a actuar en consecuencia o a exigir compensación. Esa legitimidad era en sí misma uno de los riesgos de la coerción. (Bauman, 2013, p. 10)

Sin lugar a dudas estos criterios de legitimidad son, en todo o en parte, cuestionables por el ejercicio arbitrario de la legalidad y de la ausencia de consentimiento, no solo de las víctimas de un daño ambiental, sino en la consideración de la dimensión ambiental, la importancia cultural e identidades de las comunidades que habitan un territorio y que pueden ser víctimas de un daño sea individual o colectivo. No es suficiente que estas normas sean legales, se requiere que guarden correspondencia con finalidades axiológicas o realidades sociales, con los ideales y necesidades de la comunidad y así mismo que estas disposiciones sean consentidas y avaladas no solo por los grupos gobernadores, sino por 
los grupos gobernados: "estas normas deben estar justificadas en función de creencias compartidas de los gobernantes y los gobernados" (Heywood, 2010, p. 168).

Esta concepción de legitimidad concebida a partir de creencias compartidas configura una nueva forma de legitimidad que trasciende las perspectivas de dominación y legalidad. Esa nueva forma de legitimidad posee una visión internalista, "asegurando así que se obedezca por sentido del deber antes que por temor" (Heywood, 2010, p. 169). La obediencia sería, entonces, el resultado de la aceptación, del reconocimiento del juicio de valor de la norma con los postulados internos del individuo $y$ la de sociedad sujeto a la norma.

La aceptación designa un nuevo y distinto hecho (muchos más complejos que la mera conformidad) consistentemente en que la razón del sujeto para cumplir la norma es algún tipo de identificación con el juicio de valor o la decisión que la norma implica, es decir, su internalización. En su sentido más fuerte la aceptación implicaría una identificación moral con el contenido prescriptivo de la norma de modo que el sujeto haría suya, por así decirlo, la propia norma. (Hierro, 2003, p. 17)
Esa identificación moral conllevaría a que el daño ambiental se configure como un tema perceptual, es decir, los juicios de racionalidad no solo son determinados por el arbitrio de quien legisla, sino por la valoración compensatoria de las dimensiones sociales, culturales, ideológicas y económicas, toda vez que, “el daño, está marcado por una serie de factores más sociales que jurídicos [es decir] el daño obedece más a una construcción social que a una verdadera construcción puramente jurídica o económica" (Macías, 2007, p. 147-148).

Tiene sentido que la concepción y valoración del daño no atienda solo una construcción jurídica, toda vez que dicha valoración sería arbitraria y descontextualizada. Arbitraria, en primer lugar, en el sentido de que el Estado en el ejercicio del poder de mando y a través de sus funciones legislativas no podría determinar qué configura un daño "justo", o en otras palabras qué es daño y si este está obligado a soportarse. Como lo estableció la Corte Constitucional de Colombia:

El daño al ecosistema, así ello se haga en desarrollo de una explotación lícita, desde el punto de vista constitucional, tiene el carácter de conducta antijurídica. No puede entenderse que la pre- 
via obtención del permiso, autorización o concesión del Estado signifique para su titular el otorgamiento de una franquicia para causar impunemente daños al ambiente. (Sentencia C-320 de 1998).

En segundo lugar, dicha valoración sería descontextualizada, por cuanto la determinación de valor de un daño ambiental trasciende las dimensiones ecológicas y concibe los contextos sociales y culturales, que en la mayoría de los casos son complejos por las interrelaciones e inter-conexiones que componen el contexto socio-ambiental y que conforman cosmovisiones que difieren de la lógica jurídica.

Es así como el daño ambiental, sea puro o consecutivo, genera dos valoraciones distintas, pero interdependientes: una valoración sobre el medio biofísico y una valoración territorial. Las afectaciones sobre estos dos componentes o más pueden generar otro tipo de daños: los fundamentales, los propiamente colectivos o las vulneraciones de derechos humanos. Generalmente estos tipos de daños, si bien pueden ser valorados por una población determinada, "casi en ningún caso los estudios muestran las consecuencias familiares ... el impacto de la acumulación forzada o las violaciones de derechos humanos sufridas en muchos de ellos" (Beristain, 2011, p. 129).

Por estas razones, existe una brecha entre las valoraciones del daño ambiental establecidas por el Estado y las establecidas por la sociedad. Es diferente que el legislador o legisladora lo diga, a que la sociedad lo acepte. En este caso, el criterio de legitimidad no se evoca a una decisión arbitraria del Estado, sino a un marco de consenso con la sociedad.

No es la violencia simbólica de la norma expresada a través del derecho (Bourdieu, 1987), toda vez que como lo cuestiona Rousseau “QQué es pues un derecho que perece cuando la fuerza cesa?", a lo que él mismo responde: “(...) y puesto que la fuerza no constituye derecho alguno, quedan sólo las convenciones como base de toda autoridad legítima entre los hombres". (Rousseau, 2011, p. 12). Ese tipo de convención se materializa en el consenso y en la interpretación, más que en la imposición, toda vez que es la sociedad -en el caso de un daño colectivo ambiental o una comunidad específica- la que puede considerar e interpretar el daño que está obligada a soportar.

En este sentido “...sepodríaconsiderar que el daño ambiental es el deterioro o afectación al medio ambiente que la sociedad no está dispuesta a soportar o a internalizar" (Macías, 2007, p. 148); es un daño que la sociedad legitima en correspondencia con sus cosmovisiones, culturas, ideologías y creencias y cuyo criterio de razonabilidad no se define por la formalidad de una norma en abstracto $o$ en función de un interpretación lógico formal, sino 
en las relaciones de las normas con un contexto social que destaca y abstrae los valores que componen la naturaleza esencial del ser humano.

\section{Conclusiones}

El daño ambiental, desde su naturaleza pura o consecutiva, no solo evidencia la crisis ambiental del siglo XXI, sino la crisis del Estado moderno en la consideración de la juridicidad de este mismo vía legalidad con presunción de legitimidad. Se constituye como una forma de alteración de la noción esencial de los derechos y desconoce los valores que emergen, según la naturaleza e identidad de diferentes valores culturales y sociales, que se ven en la obligación "legal", pero no "legítima" de soportar un daño, cuando la norma así lo establece.

Es incuestionable que dichas consideraciones, así resulten legales, son injustas, no por la problematización del concepto de justicia, sino porque no hay consenso; es solo una parte, el Estado el que entra a determinar ese carácter de juridicidad. Y esta determinación va mucho más allá del hacer, decir o mandar, sino del "saber". El saber cuándo se debe considerar que una comunidad determinada o la sociedad en general deben soportar un daño; el saber si es racional o no la afectación a bienes comunes y colectivos; el saber hasta qué grado la naturaleza puede superar su capacidad de resiliencia y si el equilibrio ecológico puede restaurar unas afectaciones. La valoración de un daño lo constituyen las percepciones sociales, percepciones que no solo tienen características físicas frente al impacto sobre un recurso, sino que consideran percepciones ideales que abarcan las esferas culturales, ideológicas y aun identitarias frente a las relaciones con el ambiente en general o asociado a un determinado territorio.

Esas diferentes formas de legitimación llevan a cuestionar la concepción tradicional del Estado moderno, de asociar con un mismo significado la legalidad con la legitimidad. En el contexto actual, los dos conceptos poseen características distintas, por cuanto el primero es solo una de las características del segundo. Si bien, las formas de actuar de un Estado deben consagrarse en ajuste con las formalidades legales de un ordenamiento jurídico, este tan solo constituye un primer paso; el consenso con la sociedad es el segundo, aunque dicha categorización no jerarquiza su importancia, sino el despliegue de requisitos esenciales a los que deben obedecer los criterios de racionalidad.

Ahora bien, no se puede ignorar que este consenso también posee características subjetivas, por cuanto cada comunidad es diferente y valora y percibe su relación con el medio de manera diferente y porque en sí mismo ese consenso social es o debería ser el ideal de todo Estado o forma de gobierno. Sin embargo, esta subjetividad también acompaña al Estado y a sus esferas políticas, por cuanto tiene 
que interpretar las percepciones sociales y las disposiciones legales. Este consenso siempre estará representado por partes, las cuales, aun desde el mismo marco normativo, adoptarán diferentes perspectivas frente al uso y regulación de los recursos que componen el ambiente.

\section{Referencias}

Bauman, Z. (2013). La sociedad sitiada. Argentina: Fondo de Cultura económica. $1^{\mathrm{a}} \mathrm{ed} .7^{\mathrm{a}}$ reimpresión.

Beristain, C. (2011). El derecho a la reparación en los conflictos socio-ambientales. Experiencias, aprendizajes y desafíos prácticos. Colombia: Departamento de Publicaciones Universidad Santo Tomás.

Caferrata, Néstor (2004). Introducción al derecho ambiental. México: Secretaría de Medio Ambiente y Recursos Naturales.

Fontaine, G. (2004). Enfoques conceptuales y metodológicos para una sociología de los conflictos ambientales. Recuperado de http://library.fes.de/pdf-files/ bueros/kolumbien/01993inf.htm

García, M. (2014). La eficacia simbólica del derecho. Sociología política del campo jurídico en América Latina. Colombia: IEPRI.

Gil, E. (2011). Responsabilidad extracontractual del Estado. Colombia: Editorial Temis.

González, J. (2007) La indemnización dentro de los procesos de acciones populares por daños al medio ambiente. En Daño ambiental (Tomo I, pp. 227252). Colombia: Universidad Externado de Colombia.

Hierro, L. (2003). La eficacia de las normas jurídicas. España: Editorial Ariel.

Henao, J. (1998). El daño. Análisis comparativo de la responsabilidad extracontractual del Estado en derecho colombiano y francés. Colombia: Universidad Externado de Colombia.

Henao, Juan Carlos. (2000). Responsabilidad del Estado colombiano por daños al medio ambiente. En Responsabilidad por daños al medio ambiente. Colombia: Universidad Externado de Colombia.

Heywood, A. (2010). Introducción a la teoría política. España: Ediciones Tirant Lo Blanch.

Lyotard, J. (2012). La condición postmoderna. España: Ediciones Cátedra.

Macías. (2007). El daño ambiental. Hacía una reflexión conceptual desde la filosofía y el Derecho Ambiental. En Daño ambiental. (Tomo I, pp. 125-151). Colombia: Universidad Externado de Colombia.

Noguera, P. (2004). El reencantamiento del mundo. Colombia: Universidad Nacional de Colombia.

Rousseau, J. (2011). El contrato social. México: Grupo Editorial Éxodo. 
Sousa, B. (2010). Refundación del Estado en América Latina. Perspectivas desde una epistemología del Sur. Colombia: Siglo del Hombre Editores.
Weber, Max. (1944). Economía y sociedad. México: Fondo de Cultura Económica.

Recibido: 28/3/2016 • Aceptado: 15/9/2016 